\title{
Cuantificación de la distribución geográfica del impacto económico en los eventos deportivos
}

\section{Quantification of the spatial distribution of the economic impact on sporting events \\ Jesyca Salgado-Barandela, Patricio Sanchez-Fernandez}

Facultad de Ciencias Empresariales y Turismo. Universidad de Vigo. España.

CORRESPONDENCIA:

Jesyca Salgado Barandela

j.salgado@uvigo.es

Recepción: septiembre 2020 • Aceptación: enero 2021
CÓMO CITAR EL ARTÍCULO:

Salgado-Barandela, J., \& Sanchez-Fernandez, P., (2021). Cuantificación de la distribución geográfica del impacto económico en los eventos deportivos. Cultura, Ciencia y Deporte, 16(50), 573582. http://dx.doi.org/ 10.12800/ccd.v16i50.1573

\section{Resumen}

La estimación del impacto económico de un evento deportivo requiere de una cuidada metodología para evitar la incursión en errores que sobreestimen su efecto económico. En este trabajo se cuantifica la distribución geográfica del impacto económico del Campeonato de España de Baloncesto de Clubs Infantil Masculino 2018. El evento se ha organizado en la ciudad de Marín, España (25357 habitantes), atrayendo un total de 1289 asistentes con impacto económico (809 espectadores y 480 participantes). Se trabaja con una base de datos conformada por 244 encuestas, que ha sido diseñada para identificar el gasto generado por los asistentes por zonas geográficas. Se observa que la ciudad de acogida realiza una inversión de recursos económicos y humanos en la organización del evento, pero solo obtiene un $\mathbf{2 7 \%}$ del impacto económico total. De esta manera, se identifica una fuga del impacto del $73 \%$, un $48 \%$ se dirige hacia la ciudad desarrollada más cercana y el $25 \%$ restante a zonas del alrededor. La sostenibilidad del evento para la ciudad sede está comprometida, debido a que pierde la mayor parte del beneficio económico generado por el evento. Por tanto, los posibles efectos indirectos e inducidos también se ven perjudicados.

Palabras clave: Impacto económico, efecto fuga, distribución geográfica, turismo deportivo.

\section{Abstract}

The estimation of the economic impact of a sporting event requires a careful methodology to avoid incursion into errors that overestimate its economic effect. In this work we quantify the spatial distribution of economic impact of Spanish Schools Boys Basketball Championship 2018. The event was organised in the city of Marín, Spain (25357 population), attended by a total of 1289 attendees with an economic impact (809 spectators and 480 participants). We utilise a database made up of 244 surveys, which has been designed to identify the spending generated by attendees by geographical areas. It is noticed that the host city makes an investment of economic and human resources in the organization of the event, but only gets $27 \%$ of the total economic impact. In this way, there is a leakage of $73 \%$ of the impact, $48 \%$ goes to the closest developed city and the remaining $25 \%$ of areas around the host city. The sustainability of the event for the host city is compromised, since it loses most of the economic benefit created by the event. Thus, the possible indirect and induced effects are also lessened.

Key words: Economic impact, leakage, spatial distribution, sport tourism. 


\section{Introducción}

La organización de eventos deportivos representa una oportunidad para las ciudades de desarrollar estrategias que permitan incentivar el turismo y la economía en la zona. De esta manera, los eventos deportivos se convierten en un componente clave de la estrategia de marketing de las ciudades. Como explican Kaplanidou y Vogt (2002) y Green, Costa, y Fitzgerald (2003) los eventos son una oportunidad de comercializar y promover el destino y desarrollar una imagen de marca en torno a la organización de eventos.

Según López-Carril, Añó y Villamón (2019) el desarrollo de herramientas de gestión en el deporte surge como respuesta a la demanda social y al desarrollo de una industria propia. En este sentido la medición de la repercusión económica que representan los eventos para las ciudades es un aspecto clave para ser eficaces en la consecución de los objetivos propuestos (Salgado, Barajas \& Sánchez, 2017). Una de las herramientas existentes para analizar el aspecto económico y financiero de los eventos deportivos la representan los estudios de impacto económico. Crompton (2006) define el impacto económico como el flujo económico convertido en ingresos para el territorio anfitrión, resultante del gasto atribuido a los visitantes.

La aplicación de este tipo de herramientas requiere que el analista sea especialmente cuidadoso con la metodología. Es importante ofrecer estimaciones apriorísticas y conectadas con la situación económica real del territorio. Crompton $(1995,2006)$ explica que existen once errores que se suelen cometer al realizar análisis de estudios de impacto económico. Dichos errores se pueden agrupar en cuatro bloques. En primer lugar, mal uso de los coeficientes multiplicadores. Lo que incluye: uso de las ventas en vez de los ingresos de los hogares en los multiplicadores, tergiversación de los multiplicadores de empleo, uso incremental en vez de uso normal de los coeficientes multiplicadores y uso de coeficientes multiplicadores "falseados".

En segundo lugar, la incorrecta identificación de asistentes con impacto. En este sentido es muy importante recordar que únicamente los asistentes no residentes que acuden motivados por el evento deportivo generan una inyección nueva de dinero para el territorio (Preuss, Könecke, \& Schütte, 2010). Por tanto, es necesario no solo identificar a los asistentes no residentes, sino también excluir a aquellos que no estén en el territorio motivados por el evento. Concretamente se trata de los asistentes time-switchers (aquellas personas que tenían pensado visitar el territorio igualmente y cambian la fecha para coincidir con la celebración del evento) y casuals (aquellas personas que ya estaban de visita en el territorio anfitrión y asisten al evento en vez de realizar otra actividad en la zona), definidos por Matheson (2006).

Otro aspecto vital identificado por Crompton (1995, 2006) es la definición del área de estudio con precisión. Se trataría este del primer paso antes de iniciar cualquier estudio de impacto económico. La delimitación del área geográfica de estudio influye sobre el diseño de todo el procedimiento de un estudio de impacto económico (Barajas, Salgado, \& Sánchez, 2012).

Finalmente, en cuarto lugar, se producen errores en la consideración de los costes. La omisión de determinados costes, como el gasto realizado en la construcción o reforma de infraestructuras con motivo del evento o del coste de oportunidad. En otras ocasiones el error viene determinado por incluir únicamente beneficios, ignorando por completo la existencia de costes.

Por su parte, Matheson (2006) explica que en cualquier estudio de impacto económico hay que considerar tres efectos: el efecto substitución, el efecto desplazamiento (crowding-out) y el efecto fuga (leakages). Barajas et al. (2012) explican que el efecto substitución se produce cuando el gasto realizado en el territorio no es motivado únicamente por el evento. Corresponde a la circunstancia que ocurre con los asistentes time-switchers y/o asistentes casuals. Por su parte, el efecto desplazamiento se refiere al gasto "desplazado" con motivo del evento, debido a los turistas que evitan viajar al territorio por la realización del evento. Finalmente, el efecto fuga se produce cuando la inyección de dinero inicial motivada por el evento no produce efectos indirectos e inducidos en la economía.

El efecto fuga puede generarse por diversos motivos, uno de ellos se produce cuando la ciudad sede no presenta la capacidad hotelera suficiente para albergar a los asistentes atraídos por el evento. Esto provoca que los asistentes se desplacen a las zonas de alrededor buscando los servicios y productos que necesitan. Trabajos previos, como los realizados por Connell y Page (2006), Daniels (2007), Herrero, Sanz, Devesa, Bedate, y Del Barrio (2006) y Lee, Mjelde, y Know (2013, 2017) estudian el efecto que tiene este tipo de fuga sobre la ciudad sede en el caso de eventos. De manera específica, Lee et al (2017) y Herrera et al. (2006) identifican que las ciudades vecinas de la sede del evento suelen obtener entre un 20\%-30\% del impacto económico. Por su parte, Connell y Page (2006) y Daniels (2007) observan que la ciudad más desarrollada suele obtener un mayor impacto económico en eventos deportivos con más de una sede. En general, se trata de un aspecto poco estudiado en la literatura científica, situación más apremiante cuando se trata de la organización de eventos de tamaño medio y pequeños en ciudades de tamaño pequeño. 
Por otro lado, considerando la medición del impacto económico de manera agregada, se puede observar en la literatura científica un crecimiento del interés por el análisis de los eventos de tamaño medio y pequeños. Taks, Kesenne, Chalip, y Green (2011) estudian el impacto económico del Campeonato Júnior Panamericano 2005, analizando la diferencia entre la aplicación de dos metodologías de impacto económico. Por su parte, Ramírez, Ordaz, y Rueda (2007) estiman la repercusión económica del Campeonato de Tenis Femenino de la ITF celebrado en el año 2006 en Sevilla. Los autores combinan dos herramientas de impacto económico para estimar el efecto directo, indirecto e inducido producido por el evento, mientras que Gibson, Kaplanidou, y Kang (2012) estudian la repercusión económica, social y ambiental de seis eventos deportivos de tamaño medio. De esta manera, es posible considerar estudios que identifican una repercusión positiva de la organización de este tipo de eventos. Como explican Ziakas y Costa (2011a, 2011b), los eventos de tamaño medio son más sostenibles, fáciles de gestionar y suponen una menor inversión para las ciudades.

De forma específica, en la modalidad deportiva del baloncesto se pueden identificar diversos estudios de investigación sobre la repercusión económica de los eventos existentes. Un ejemplo se observa en el estudio desarrollado por Balciunas, Jasinskas y Koisova (2014), donde se estima el impacto directo e indirecto en la economía del Eurobasket 2011, además de exponer las posibilidades de mejora del evento para futuras ocasiones. Por su parte, Propheter (2012) analiza el impacto generado por la construcción de los estadios profesionales de baloncesto y su función como catalizadores del desarrollo económico. Otro ejemplo para destacar es el estudio llevado a cabo por Pujol y García-del-Barrio (2006), en el que estiman el valor mediático del Mundial de Basket 2006. A nivel local, Antelo, Barajas, Salgado y Sánchez (2014) realizaron el estudio de impacto económico del equipo Monbus Obradoiro, participante de la Liga Endesa (primera división del baloncesto en España).

Considerando lo previamente explicado, el objetivo de este trabajo se centra en cuantificar la distribución geográfica del impacto económico del Campeonato de España de Baloncesto de Clubs Infantil Masculino realizado en la ciudad de Marín, entre los días 10 y 16 de junio de 2018. Para ello se estima el gasto realizado por los asistentes con impacto económico clasificado por zonas geográficas. De esta manera se identifica una fuga del impacto económico motivada por una capacidad hotelera limitada que desplaza a los asistentes a las zonas de alrededor.
La primera parte del artículo se centra en una revisión de la literatura que aborda las limitaciones existentes en la medición del impacto económico, así como los estudios que se centran en la medición del impacto económico de eventos deportivos de tamaño medio tanto a nivel agregado, como aquellos que miden la distribución geográfica del impacto. En segundo lugar, se explica el enfoque metodológico y la base de datos empleada para la consecución del objetivo planteado. A continuación, se describen los resultados y hallazgos obtenidos mediante el análisis empírico. Finalmente, los apartados de discusión y conclusiones permiten analizar los resultados obtenidos en función de los trabajos previos existentes y aportar posibles soluciones a la problemática identificada.

\section{Metodología}

\section{Aspectos metodológicos}

No existe una única metodología para estimar la repercusión económica de los eventos. Como explican Sánchez, Salgado, Rodríguez y Barajas (2016) y Barajas et al. (2012), las herramientas existentes no son excluyentes entre sí y pueden combinarse para mejorar la capacidad de estimación de los resultados.

De forma específica para el estudio que se lleva a cabo se toma como referencia el método Análisis Coste-Beneficio (ACB). Como explica Késenne (2005), se trata de una metodología que permite comparar el beneficio de los eventos deportivos para una región o país con los costes de los factores de producción necesarios para organizar el evento.

La utilización de esta herramienta es habitual en los estudios sobre eventos deportivos de tamaño medio. Autores como Buning, Cole y McNamee (2016) y Taks, et al. (2011) defienden la estimación de la inyección inicial de dinero para el caso de los eventos de tamaño medio, frente al uso de las tablas Input-Output. Crompton (1995, 2006), Howard y Crompton (2005) y Matheson (2009) explican que el uso de las Tablas Input-Output para realizar mediciones en el caso de eventos deportivos puede llevar a la sobreestimación de los resultados si no se aplica de manera adecuada. Barajas et al. (2012) indican que su empleo es más adecuado para eventos de gran nivel que se celebren de forma regular y tienen una larga duración.

La aplicación del Análisis Coste-Beneficio permite la estimación del impacto económico directo, no se obtiene impacto indirecto, ni inducido. Para la estimación del impacto indirecto e inducido sería necesario aplicar los multiplicadores a partir de las Tablas 
Input-Output. En el caso objeto de estudio esto no es posible debido a que las Tablas Input-Output de Galicia no se encuentran territorializadas, ni desglosan la partida correspondiente a deporte. Además, como se ha explicado de manera previa, en el caso de los eventos de tamaño pequeño y con una duración puntual no se recomienda su utilización para evitar una sobreestimación del resultado del impacto.

El desarrollo de la metodología consta de un procedimiento compuesto por tres fases. En la primera fase se identifican los costes y beneficios que el evento supone para la ciudad sede, diferenciando cuáles de los mismos generan una inyección nueva de dinero para el territorio, así como las salidas de dinero generadas por el evento. En segundo lugar, los costes y beneficios deben ser cuantificados en unidades monetarias. Finalmente, en la tercera fase se estima el impacto directo mediante la diferencia entre los costes y beneficios identificados.

En relación a la distribución geográfica del impacto y la estimación del efecto fuga se sigue el trabajo desarrollado por Daniels (2007), que emplea un diseño de encuesta que permite identificar la distribución geográfica del impacto económico. Para determinar la distribución geográfica se considera en primer lugar la ciudad cercana más desarrollada, que corresponde a Pontevedra (con 82.802 habitantes), ubicada al norte de la ciudad de Marín, a $7.5 \mathrm{~km}$. Se incluyen también los ayuntamientos que forman parte de la comarca de Pontevedra: Poio, Barro, Campo Lameiro, La Lama, Puentecaldelas y Vilaboa, ubicados en un radio de 9.8 $\mathrm{km}$ de Marín. Además, se incluyen los tres ayuntamientos que se encuentran al sur de Marín, conformados por Cangas, Bueu y Moaña, ubicados en un radio de $9.4 \mathrm{~km}$. Una vez determinada el área de la distribución geográfica se clasifica en tres zonas:

- Ciudad de Marín: que organiza y es sede del evento.

- Comarca de Pontevedra: zona más cercana a la sede del evento, que además representa el núcleo urbano más desarrollado. Conformada por la ciudad de Pontevedra y los ayuntamientos de Poio, Barro, Campo Lameiro, La Lama, Puentecaldelas y Vilaboa.

- Resto de zonas: localidades que pertenecen a otros ayuntamientos cercanos donde también se hospedan asistentas al evento. Conformado por los ayuntamientos de Cangas, Bueu y Moaña.

De esta manera, en la realización de la encuesta es necesario preguntar de forma específica qué parte del gasto de los asistentes se realiza en Marín y qué parte en alguna de las zonas establecidas. Esto va a permitir realizar una correcta distribución geográfica del gasto del evento.

\section{Técnica de obtención de datos}

La información necesaria para la realización del estudio sobre el impacto económico de la organización del Campeonato de España de Baloncesto de Clubes Infantil Masculino se consiguió mediante la realización de una encuesta a los asistentes. La encuesta empleada ha sido previamente validada por profesionales expertos en el ámbito estadístico y de la economía del deporte. Además, dicha encuesta se ha aplicado de forma previa con éxito en diferentes eventos deportivos. De esta manera, se cuenta con una plantilla base que se adapta a las necesidades específicas de la información a obtener en el evento. Por otra parte, la entidad organizadora facilitó la información relacionada con los gastos e ingresos motivados por el evento.

Las encuestas se llevan a cabo durante un total de tres días. El 85\% de las mismas se realiza en los dos primeros días del campeonato, ya que al inicio coinciden todos los equipos participantes y los espectadores que acuden a ver los partidos. A medida que van descalificando equipos, el volumen de participantes y espectadores baja. El 15\% restante de las encuestas se lleva a cabo durante la final del campeonato, con la intención de identificar la llegada de nuevos asistentes, que acuden directamente a ese día del evento.

En total, se encuestaron 244 personas (considerando una población de 600 personas) lo que implica una margen de error del $5 \%$ con un nivel de confianza del 95\%. Las estimaciones previas de asistencia alcanzan las 1200 personas en total, pero dicha cantidad no representa la población susceptible de ser encuestada. La mayoría de los asistentes acuden acompañados (miembros del mismo núcleo familiar), y por las características de la encuesta solo se debe realizar a un miembro (siempre mayor de edad) de cada grupo que acude junto. En la encuesta se pide el gasto total realizado en la ciudad con motivo del evento por toda la unidad familiar (en caso de acudir acompañado). Si se realiza la encuesta a distintos miembros del mismo grupo en momentos diferentes del evento los datos obtenidos darán una falsa realidad de mayor número de asistentes. Por este motivo para la estimación de la muestra se emplea la mitad de la población total; en este caso, 600 personas. Para garantizar la aleatoriedad de la encuesta, los encuestadores siguieron un patrón de conducta dirigiéndose a una persona de cada tres en cada zona y realizándolas en diversos momentos a lo largo de las jornadas. El texto de la encuesta a los espectadores se estructura en cuatro partes diferenciadas con un total de 24 preguntas, que se describen a continuación. 
La primera parte del cuestionario aborda la identificación en términos deportivos de la persona encuestada, entre las que destaca su condición de participante en la prueba o solo espectador y, en caso de que participe, la selección autonómica de pertenencia. Además, en esta primera fase también se recoge si la persona encuestada es residente en el área de Marín o no; en caso de que la respuesta sea negativa, se recogerá el lugar de origen.

La segunda parte recoge preguntas relacionadas a la planificación del evento por parte de los asistentes, si acuden con motivo del evento, dónde pasaron la noche y cuántos días, si acudieron al evento en años anteriores o qué actividades realizaron. De manera concreta, en esta parte de la encuesta se identifican a los asistentes casuals y time-switchers, que ya han sido definidos previamente. Este tipo de asistentes no se debe considerar como personas con impacto ya que no están generando un gasto autónomo, iban acudir a la ciudad de todas maneras, se realizara o no el evento. Para calcular el porcentaje de asistentes casuals y timeswitchers se pregunta si asisten a la ciudad exclusivamente con motivo del evento. A aquellos que respondían que no se les preguntaba a continuación si habían pensado acudir en otras fechas pero fue la celebración del evento lo que los hizo cambiar de plan o si están en la ciudad por otros motivos.

En este apartado también se incluyen los aspectos económicos relacionados con los asistentes. Concretamente se pregunta sobre la cantidad de dinero que tenía intención de gastar en la ciudad y su reparto en función de las zonas de impacto establecidas (ciudad de Marín, comarca de Pontevedra y resto de zonas). Dado que la experiencia dice que una pregunta tan directa resulta difícil de contestar, se optó por proponer tres tipos de preguntas diferenciadas para poder tratar los datos de la manera más rigurosa posible. Se preguntaban por orden y, si no se obtenía respuesta, se ofrecía la siguiente como alternativa.

La primera de ellas analiza el gasto efectuado por conceptos. Si el encuestado no es capaz (o no quiere) distribuir el gasto, se le ofrece la opción de indicar el montante global del gasto, y si aun así tampoco responde, se facilita un rango de intervalos entre los que estima que se situaría su gasto. Para la obtención del gasto medio realizado, en primer lugar se calcula la media de cada concepto de gasto y se suma obteniendo el total de gasto medio por conceptos. En segundo lugar, se calcula la media de gasto de aquellos encuestados que indicaron una cantidad global. A continuación, se establecen marcas de clase que se multiplican por la frecuencia (número de personas que responde en cada intervalo) para obtener el gasto medio medido por intervalos. Finalmente, para calcular el gasto medio total se realiza un promedio ponderado de los tres tipos de gasto considerando el número de respuestas en cada caso.

La tercera parte se enfocaba a conocer la vertiente turística de los encuestados. Se incluye, puesto que, aunque no es cuantificable, sirve de orientación sobre su posible incidencia futura en el turismo con su consecuente rendimiento económico. Así, se preguntaba si vinieron en años anteriores, si volverían en años posteriores, la puntuación sobre la imagen de la ciudad para el evento y la comparación de este con otros similares a los que habían asistido, si estaban satisfechos con la organización del evento y si eran seguidores de eventos deportivos.

La cuarta parte recoge aspectos socio-demográficos de las personas encuestadas, tales como edad, sexo, profesión, nivel educativo o el tramo de ingresos individuales anuales. También resulta de interés en este apartado preguntar por el número de acompañantes.

Finalmente, una vez recogida toda la información procedente de las encuestas se procesó para el posterior análisis de los datos.

\section{Resultados}

\section{Estimación del impacto económico directo de los participantes}

Como se extrae de la lista de inscritos oficial, acuden al campeonato 32 equipos de las distintas selecciones autonómicas. Cada equipo contaba con 12 jugadores y 3 técnicos. Dichas selecciones conformaban un total de 480 participantes.

El gasto de los participantes es gestionado por los distintos equipos. Para conocer el gasto realizado se cuenta con la información facilitada por una central de reservas que gestionó el hospedaje de 25 de los 32 equipos participantes, 19 equipos se hospedaron en la comarca de Pontevedra, generando un gasto de 45000 euros, mientras que 6 equipos se hospedaron en Marín, generando un gasto de 7500 euros. En todos los casos la estadía se realiza en régimen de pensión completa.

Con respeto a los siete equipos restantes, los dos provenientes de Santiago de Compostela, no pasan la noche en Marín o comarca, dada la cercanía del lugar de procedencia con la sede del Campeonato. Por último, existen 5 equipos que se hospedan en el área definida como "resto de zonas", que no pueden ser incluidos en el impacto porque no se tienen datos sobre el gasto realizado durante su estadía. 


\section{Estimación del impacto económico directo de los} espectadores

Como se explica de forma previa, se ofrecen tres posibilidades de pregunta para obtener el gasto realizado por los espectadores en la ciudad con motivo del evento. Debido a que se identifica el gasto por zonas geográficas, se pide a los encuestados que no pernoctan en Marín que especifiquen si realizan algún gasto en la ciudad.

Un total de 138 personas contestaron analizando por conceptos el gasto que estimaban que iban a realizar. El importe medio de gasto que habían previsto realizar es de 316.6 euros. Otras 72 personas contestaron con la cifra global estimada de su gasto en la ciudad y 18 respondieron a la pregunta de intervalo de gasto. En estos dos últimos casos, el importe medio resultante fue de 222.2 euros y 186.1 euros, respectivamente.

Para determinar el gasto del grupo de personas que contestó dando importes por tramos, se utilizan las marcas de clase. Como se explicó en el apartado metodológico, las marcas de clase (punto medio de cada intervalo de gasto) se multiplican por la frecuencia (número de personas que contestaron en ese intervalo). La Tabla 1 muestra los resultados del cálculo del gasto por intervalos.

Para el cálculo del gasto medio por persona se realiza un promedio ponderado del gasto obtenido en función de cada grupo de respuestas. En la tabla 2 se puede observar que el resultado obtenido de la media ponderada es de 276.5 euros. Este dato de gasto se emplea para realizar la estimación del impacto de los espectadores. Gracias a ofrecerse diversas alternativas para contestar, se obtuvo un grado conjunto de respuesta del 93.4\%, lo que garantiza la representatividad estadística, al tiempo que puede ser considerado como un porcentaje de respuesta altamente satisfactoria para un estudio como el aquí llevado a cabo.

A partir de la encuesta realizada se determina que el $95.7 \%$ de los espectadores que asisten son no residentes, frente a un $4.3 \%$ que proceden de Marín. También se identifica que no hay existencia de espectadores casuals y time-switchers. Estos porcentajes se emplean para calcular el número de espectadores con impacto. Dado que la entrada al evento fue gratuita, el número de espectadores se obtuvo realizando un recuento en los días del evento. De este modo, el número total ascendió a 845 personas. Para realizar dicha estimación se cuenta en cada partido del primer día del evento el número de espectadores. Esta forma de estimar es posible, puesto que en cada partido el público estaba conformado por las familiares y/o acompañantes de los jugadores, que llegaban para ver dicho partido y se
Tabla 1. Cálculo de gastos para las respuestas por intervalos.

\begin{tabular}{lccc}
\hline $\begin{array}{l}\text { Intervalo de } \\
\text { gasto }(€)\end{array}$ & $\begin{array}{c}\text { Marca de clase } \\
(\mathbf{x i})\end{array}$ & $\begin{array}{c}\text { Frecuencia } \\
(\mathbf{f i})\end{array}$ & $\mathbf{x i}^{*} \mathbf{f i}$ \\
\hline $0-100$ & $50 €$ & 5 & $250 €$ \\
$100-300$ & $200 €$ & 11 & $2200 €$ \\
$300-600$ & $450 €$ & 2 & $900 €$ \\
Más de 600 & - & 0 & - \\
Total & & 18 & $3350 €$ \\
\hline
\end{tabular}

Fuente: Elaboración propia a partir de las encuestas.

Tabla 2. Cálculo de la media ponderada de gasto por persona.

\begin{tabular}{lccc}
\hline $\begin{array}{l}\text { Tipo de } \\
\text { pregunta }\end{array}$ & $\begin{array}{c}\text { Importe } \\
\text { del gasto }\end{array}$ & Personas & $\begin{array}{c}\text { Media de gasto } \\
\text { por persona }\end{array}$ \\
\hline $\begin{array}{l}\text { Gasto por } \\
\text { conceptos }\end{array}$ & $43687.28 €$ & 138 & $316.6 €$ \\
$\begin{array}{l}\text { Cantidad total } \\
\text { de gasto }\end{array}$ & $15998.66 €$ & 72 & $222.2 €$ \\
$\begin{array}{l}\text { Intervalos de } \\
\text { gasto }\end{array}$ & $3350.00 €$ & 18 & $186.1 €$ \\
Media ponderada & & & $276.5 €$ \\
\hline
\end{tabular}

Fuente: Elaboración propia a partir de las encuestas.

Tabla 3. Impacto económico de los espectadores.

\begin{tabular}{lccc}
\hline $\begin{array}{l}\text { Zona de } \\
\text { impacto }\end{array}$ & $\begin{array}{c}\text { Personas } \\
\text { con impacto }\end{array}$ & $\begin{array}{c}\text { Gasto medio } \\
\text { por persona }\end{array}$ & $\begin{array}{c}\text { Impacto } \\
\text { Económico }\end{array}$ \\
\hline Marín & 282 & $276.5 €$ & $77973 €$ \\
$\begin{array}{l}\text { Comarca de } \\
\text { Pontevedra }\end{array}$ & 292 & $276.5 €$ & $80738 €$ \\
Resto de zonas & 235 & $276.5 €$ & $64977.5 €$ \\
\hline
\end{tabular}

Fuente: elaboración propia a partir de las encuestas.

marchaban de la instalación una vez que este finalizaba. Por otra parte, también facilitó esta forma de recuento el número de espectadores a cada partido, que no superaron a las 65 personas de promedio. Del total de espectadores estimados es necesario deducir los residentes, que tienen una representación del $4.31 \%$, sumando así 36 espectadores residentes. De esta manera, el número de espectadores con impacto es de 809.

Una vez determinados los espectadores que van a ocasionar una repercusión económica en la ciudad, se multiplica esa cifra por el gasto medio ponderado por persona indicándose la repercusión sobre Marín, la comarca de Pontevedra y el resto de las zonas (ver Tabla 3). Se emplea el número de personas con impacto que se alojan en las tres áreas establecidas, como se explicó en el apartado anterior.

\section{Estimación del impacto económico directo de la organización}

Para efectuar la estimación directa del impacto económico se preguntó a la Organización sobre los gastos en que incurrieron para celebrar el Campeona- 
to, y qué ingresos obtuvieron para financiarlo. En este caso se identifican tres entidades que participan en la organización. Por una parte, el Ayuntamiento de Marín y la Federación Española de Baloncesto (FEB), que promueven y organizan el evento, y, por otra, el equipo Peixe Galego, que es un colaborador técnico, aportando 45 voluntarios para el desarrollo del campeonato. A partir de estos datos se determinaron los efectos negativos y positivos que tuvo la celebración del Campeonato para el territorio.

La FEB cubre el hospedaje y mantenimiento de los 20 árbitros y 5 técnicos arbitrales, desde el inicio del campeonato hasta cuartos de final, lo que genera un gasto de 4600 euros, que revierte directamente sobre la ciudad sede. Este gasto representa una entrada de dinero nuevo para la economía local. Para el resto del Campeonato dicho gasto es asumido por el Ayuntamiento, lo que se explica a continuación.

El Ayuntamiento de Marín realiza una serie de gastos con motivo del evento, de los cuales 18438.55 euros revierten directamente en la economía local. Dicha cantidad no puede considerarse como impacto económico puesto que no representa dinero nuevo. Se produce lo que se denomina como recirculación del dinero, ya que se trata de recursos que ya estaban en la ciudad. De esta manera, estos gastos ni suman ni restan para el impacto.

El impacto económico negativo proviene de los gastos que la organización realiza fuera de la ciudad de Marín. En este caso se trata de gastos que asume el Ayuntamiento de Marín y que corresponden al pago del canon del evento, los servicios médicos, publicidad y derechos arbitrales. Dichos gastos suman un impacto económico negativo de 21218.20.

Los ingresos con repercusión en la ciudad de Marín, que corresponden a los gastos realizados por la FEB, aminoran el impacto de los gastos realizados por la organización fuera de la ciudad. Como se puede observar, los gastos en los que incurre el Ayuntamiento fuera fueron mayores que los ingresos percibidos por la misma razón. Por lo tanto, suponen una reducción neta de recursos de 16618.20 euros.

\section{Impacto económico directo total del evento y su distribución geográfica}

A partir del impacto directo generado por los participantes, espectadores y la organización se puede obtener el impacto directo total del evento. En el caso de estudio se observa que la repercusión generada por los espectadores y participantes es positiva, mientras que la actividad generada por la organización implica un impacto negativo (ver Tabla 4). Como se ha explicado
Tabla 4. Impacto económico directo total del evento.

\begin{tabular}{lc}
\hline Impacto por zona geográfica & Impacto en euros \\
\hline Ciudad de Marín & \\
Participantes & $7500 €$ \\
Espectadores & $77973 €$ \\
Organización & $-16618.2 €$ \\
Total & $68854.8 €$ \\
\hline Comarca de Pontevedra & \\
Participantes & $45000 €$ \\
Espectadores & $80738 €$ \\
Organización & $0.00 €$ \\
Total & $125738 €$ \\
\hline Resto de zonas & \\
Participantes & $0.00 €$ \\
Espectadores & $64977.5 €$ \\
Organización & $0.00 €$ \\
Total & $64977.5 €$ \\
\hline
\end{tabular}

Fuente: elaboración propia a partir de las encuestas.

de forma previa, no se conoce el dato del gasto realizado por los participantes que se hospedan en otras zonas, por este motivo aparece como cero, tal como se muestra en la tabla 4.

Se observa que la ciudad que organiza el evento no obtiene la totalidad de la repercusión económica debido principalmente a una limitación en la capacidad hotelera. Un total de 527 personas y 24 equipos no se alojan en la ciudad de Marín. Además, la ciudad asume la totalidad de los gastos mientras que la comarca de Pontevedra y otras zonas de alrededor obtienen beneficios sin haber consumido recursos ni esfuerzo. La sede del evento soporta una fuga de impacto directo de 190715.5 euros, lo que representa el $73 \%$ del impacto generado por la organización del evento. La ciudad desarrollada más cercana (Pontevedra) es la más beneficiada, obteniendo un $48 \%$ del impacto, mientras que pequeñas zonas de los alrededores a Marín obtienen un $25 \%$ y en la ciudad sede únicamente repercute un $27 \%$ del impacto directo.

\section{Discusión}

Los resultados obtenidos permiten identificar la obtención de un impacto económico positivo para la ciudad por la organización del evento. Sin embargo, al cuantificar la distribución geográfica del impacto se identifica una importante limitación referida al efecto fuga. En la ciudad sede únicamente repercute el $27 \%$ del impacto directo generado por el evento, mientras que el $73 \%$ restante se fuga a las zonas de alrededor. El primer hallazgo importante se centra en los porcentajes de fuga obtenidos. Los trabajos previos desarrollados por Lee et al. (2017) y Herrera et al. (2006) 
establecen la fuga de dinero hacía otros territorios entorno al 20\%-30\% del total de impacto económico. Esto es debido a que el evento se organizaba en el núcleo urbano más desarrollado. En el caso de estudio, la ciudad sede del evento tiene cerca una ciudad más desarrollada que está arrastrando la mayoría del impacto económico, generando una fuga del $73 \%$ del impacto.

El segundo hallazgo relevante corrobora y amplía los resultados de Connell y Page (2006) y Daniels (2007). Estos autores indican que las ciudades más desarrolladas obtienen un mayor impacto económico cuando se organiza un evento con más de una sede. De esta manera, nuestro trabajo permite demostrar que esta situación se hace más tangible cuando el evento se organiza en una ciudad pequeña con respecto a la ciudad cercana más desarrollada.

Por otro lado, hay que considerar que la ciudad se plantea el desarrollo de una estrategia de promoción turística basada en el turismo deportivo, por lo que organiza numerosos eventos deportivos a lo largo del año. En este sentido, la medición de la distribución geográfica del impacto ha permitido identificar que la estrategia planteada presenta claras deficiencias y no es sostenible a largo plazo. La ciudad sede invierte recursos económicos y humanos en la organización de eventos, obteniendo un retorno mínimo en relación a la repercusión económica. Además, al producirse un efecto fuga los efectos indirectos e inducidos se verán afectados en la misma medida. La ausencia de una correcta planificación estratégica en la atracción y organización de los eventos deportivos es una circunstancia que ha sido reflejada por distintos autores, como es el caso de Ziakas (2019) o Antchak (2017).

Se identifican además otros aspectos relacionados con el impacto del evento susceptible de discusión. Se observa que el motor de la repercusión económica en el Campeonato de España de Baloncesto Infantil Masculino son los asistentes. Dentro de este grupo, los espectadores representan un mayor impacto económico, debido principalmente a que su gasto medio es más elevado que el realizado por los participantes. Los espectadores tienen un impacto económico sobre Marín de 77973 euros, de 80738 sobre la comarca de Pontevedra y de 64978 sobre el resto de zonas, mientras que el impacto de los participantes es de 7500 euros sobre Marín y 45000 euros sobre la comarca de Pontevedra. Estudios previos, como los realizados por Gibson et al. (2012), Salgado, Sánchez, Pérez y Barajas (2018a) y Sánchez, Salgado, Rodríguez y Barajas (2016) corroboran estos resultados al identificarse que en campeonatos con similares características los espectadores generan la mayor proporción del impacto económico.
En relación a la media de gasto, dos estudios anteriores desarrollados por Salgado et al. (2018a) y Sánchez et al. (2016) sobre eventos organizados en la ciudad de Pontevedra (ciudad colindante con Marín) permiten identificar medias de gasto similares. Se trata del caso del Campeonato Máster de Natación (278 euros de gasto medio por persona) y del Campeonato Absoluto de Natación (260 euros de gasto medio por persona), mostrando así una media de gasto similar a la presentada por el campeonato objeto de estudio, que alcanzó los 276 euros. Es importante destacar que en la media de gasto de los turistas deportivos también influyen aspectos de tipo demográficos y las características deportivas del evento. Los trabajos sobre determinantes de gasto desarrollados por Brida, Schubert, Osti y Barquet (2011), Saayman y Saayman (2012), Salgado, Barajas y Sánchez (2018b), Salgado, Sánchez, y Barajas (2020) y Sato, Jordan, Kaplanidou y Funk (2014) han obtenido hallazgos significativos al respecto.

El impacto generado por las entidades que organizan el evento es negativo. El Ayuntamiento de Marín cede el uso de las instalaciones, paga un canon y cubre una serie de gastos directamente relacionados con la gestión del evento. Por otra parte, la Federación Española de Baloncesto (FEB) cubre los gastos de hospedaje y mantenimiento de los árbitros y técnicos arbitrales durante los cinco primeros días del evento. De esta manera, los gastos realizados por el Ayuntamiento fuera de la ciudad con motivo del evento (21218 €), superan a los gastos realizados por la FEB en Marín (4600 €). Con todo, se ve compensada por la repercusión económica positiva de los asistentes. En los trabajos previos de Salgado et al. (2018a) y Sánchez et al. (2016) también se puede identificar que la actividad económica generada por la organización es negativa. Esto quiere decir que las salidas de dinero fuera de la ciudad de acogida han sido mayores que las entradas de dinero nuevo. Esta situación, en el caso de la entidad que organiza el evento, puede ser habitual, ya que hay determinados gastos que la organización no puede evitar realizar fuera de la ciudad.

Se pueden acotar en dos los pilares fundamentales que permiten la generación de impacto económico positivo en el evento analizado. En primer lugar, el hecho de no tener que realizar inversiones específicas en la celebración del evento. En el caso analizado se aprovechan las infraestructuras existentes y no se incurre en la construcción o reforma de instalaciones. Este se muestra como un aspecto vital señalado por autores como Duglio y Beltramo (2017), Malchrowicz-Mósko y Póczta (2018) y Salgado et al. (2018a) para asegurar la sostenibilidad de los eventos de tamaño medio y pequeño. En segundo lugar, un aspec- 
to de valiosa importancia lo representan la atracción de turistas con motivo del evento. En el caso de estudio se trata específicamente de turistas deportivos, ya que el motivo principal de su asistencia es el campeonato. Dicho de otra manera, si no se hubiera celebrado el evento en Marín esas personas no acudirían a la ciudad (con casi toda seguridad estarían allí donde se celebrara) y, por lo tanto, no tendrían la oportunidad de visitar tanto la ciudad como el entorno. En suma, el evento atrajo más de 1200 turistas deportivos con el motivo del evento. Igualmente es importante destacar que el evento tiene una duración de 7 días, lo que aumenta el tiempo de estancia de los asistentes y, por lo tanto, el gasto medio. De esta manera, los asistentes pasan un promedio de 4.5 días en la zona con motivo del Campeonato. Como se analiza en los trabajos de Csobán y Serra (2014), Salgado et al. (2020) y Taks, Kesenne, Chalip y Green (2009) los asistentes al evento, además de los gastos en alojamiento y manutención, pueden realizar otro tipo de actividades como visitas turísticas y/o culturales, compras o actividades gastronómicas, que aumentan la inyección de dinero para la ciudad de acogida.

Finalmente, es importante el planteamiento de acciones que puedan disminuir el efecto fuga producido. La consideración de un aumento en la capacidad hotelera no suele ser una acción viable para el caso de ciudades pequeñas. En este sentido, las acciones tienen que ir dirigidas a aumentar la cooperación y coordinación entre las ciudades (Smith, 2010). De tal forma que se trabaje en una cartera de eventos anual que permita el aprovechamiento de las distintas instalaciones existentes en las ciudades para mantener durante todo el año el gasto turístico activo. Además, la existencia de coordinación entre ciudades evitará el solapamiento de los eventos y por lo tanto que los negocios de alojamiento y restauración se encuentren con fechas saturadas y en otros momentos totalmente vacíos.

\section{Conclusiones}

El trabajo llevado a cabo permite cuantificar la distribución geográfica de la repercusión económica por la organización del Campeonato de España de Baloncesto de Clubes Infantil Masculino en una ciudad de tamaño pequeño. Se establecen tres zonas de distribución geográfica del impacto económico, identificando una fuga del $73 \%$ de la repercusión económica del impacto de la ciudad sede a las zonas de alrededor. El $48 \%$ de la fuga del impacto se dirige a la ciudad cercana más desarrollada. Los resultados obtenidos implican una baja sostenibilidad y poca eficiencia en la estrategia de la organización de eventos de la ciudad sede. La medición del impacto económico se muestra así como una herramienta útil en la medición de la sostenibilidad del evento para los territorios de acogida. Aún son escasos los estudios que profundizan en este tipo de análisis, centrándose únicamente en los resultados agregados. La investigación futura debe incidir en la medición de los aspectos que limiten la repercusión económica, como es el caso del efecto fuga. Esto va a permitir un mejor desarrollo de estrategias y una asignación de recursos más eficiente.

\section{B I B LIO G R A FÍ A}

Antchak, V. (2017). Portfolio of major events in Auckland: Characteristics, perspectives and issues. Journal of Policy Research in Tourism, Leisure and Events, 9(3), 280-297. https://doi.org/10.1080/1940796 3.2017.1312421.

Antelo, X., Barajas, A., Salgado, J., \& Sánchez, P. (2014). Estudio de impacto socioeconómico y análisis de impacto social Rio Natura Monbus Obradoiro SAD. Universidad de Vigo. http://abarajas.webs.uvigo.es/ estudio\%20de\%20impacto\%20economico\%20(Solo).pdf.

Balciunas, M., Jasinskas, E., \& Koisova, E. (2014). Economic contribution of sports event: Analysis of Eurobsasket 2011 example. Transformations in Business and Economics, 13(2), 41-54.

Barajas, A., Salgado, J., \& Sánchez, P. (2012). Problemática de los estudios de impacto económico de eventos deportivos. Estudios de Economía Aplicada, 30 (2), 441-462.

Brida, J.G., Schubert, S., Osti, L., \& Barquet, A. (2011). An analysis of tourists'expen- diture on Winter sports events through the Tobit censorate model. Tourism Economics, 17(6), 1197-1217. http://dx.doi. org/10.2139/ssrn.1547694.

Buning, R. J., Cole, Z. D., \& McNamee, J. B. (2016). Visitor expenditure within a mountain bike event portfolio: Determinants, outcomes, and variations. Journal of Sport \& Tourism, 20(2), 103-122. https://doi. org/10.1080/14775085.2016.1239547.
Connell, J., \& Page, S. J. (2005). Evaluating the economic and spatial effects of an event: the case of the world medical and health games. Tourism Geographies, 7(1), 63-85. https://doi.org/10.1080/ 1461668042000324067.

Crompton, J.L. (1995). Economic Impact Analysis of Sports Facilities and Events: Eleven Sources of Misapplication. Journal of sport Management, 9, 15-35. https://doi.org/10.1123/jsm.9.1.14.

Crompton, J.L. (2006). Economic Impact Studies: Instruments for Political Shenanigans?. Journal of Travel Research, 45, 67-82. https://doi. org/10.1177/0047287506288870.

Csobán, K. V., \& Serra, G. (2014). The role of small-scale sports events in developing sustainable sport tourism-a case study of fencing. Applied Studies in Agribusiness and Commerce, 8(4), 17-22. https://ideas.repec. org/a/ags/apstra/210470.html.

Daniels, M.J. (2007). Central place theory and sport tourism impacts. Annals of Tourism Research 34 (2), 332-347. https://doi.org/10.1016/j. annals.2006.09.004.

Duglio, S., \& Beltramo, R. (2017). Estimating the economic impacts of a small-scale sport tourism event: The case of the Italo-Swiss mountain trail CollonTrek. Sustainability, 9(3), 343. https://doi.org/10.3390/ su9030343.

Gibson, H.J., Kaplanidou, K., \& Kang, S.J. (2012). Small-scale event 
sport tourism: A case study in sustainable tourism. Sport management review, 15(2): 160-170. https://doi.org/10.1016/j.smr.2011.08.013.

Green, B. C., Costa, C., \& Fitzgerald, M. (2003). Marketing the Host City: Analyzing Exposure Generated by a Sport Event. International Journal of Sports Marketing \& Sponsorship, 4, 48-66. https://doi.org/10.1108/ IJSMS-04-04-2003-B005.

Herrero, L. C., Sanz, J. Á., Devesa, M., Bedate, A., \& Del Barrio, M. J. (2006). The economic impact of cultural events: a case-study of Salamanca 2002, European Capital of Culture. European urban and regional studies, 13(1), 41-57. https://doi.org/10.1177/0969776406058946.

Howard, D. y Crompton, J. (2005). Financing Sports 2nd Edition, Fitness Information Tecnology, Morgantown.

Kaplanidou, K., \& Vogt, C. (2007). The interrelationship between Sport Event Destination Image and Sport Tourists' Behaviours. Journal of Sport\& Tourism, 12(3-4), 183-206. doi:10.1080/14775080701736932.

Késenne, S. (2005). Do We Need an Economic Impact Study or a Cost-Benefit Analysis of a Sports Event? European Sport Management Quartely, 5 (2), 133-142. https://doi.org/10.1080/16184740500188789.

Lee, C.K., Mjelde, J.W., \& Kwon, Y.J. (2017). Estimating the economic impact of a mega-event on host and neighbouring regions. Leisure Studies, 36 (1), 138-152. https://doi.org/10.1080/02614367.2015.1040828.

López-Carril, S., Añó, V., \& Villamón, M. (2019). El campo académico de la Gestión del Deporte: pasado, presente y futuro. Cultura, Ciencias y Deporte, 14(42), 277-287.

Malchrowicz-Mośko, E., \& Poczta, J. (2018). A Small-Scale Event and a Big Impact-Is This Relationship Possible in the World of Sport? The Meaning of Heritage Sporting Events for Sustainable Development of Tourism-Experiences from Poland. Sustainability, 10(11), 4289. https://doi.org/10.3390/su10114289

Matheson, V. (2009). Economic Multpliers and Mega-Event Analysis. International Journal of Sport Finance, 4, 63-70. https://crossworks. holycross.edu/econ_working_papers/104/.

Matheson, V. A. (2006). Mega-Events: The effect of the world's biggest sporting events on local, regional, and national economies. Massachusetts: Department of Economics, College of the Holy Cross. Recuperado de: https://crossworks.holycross.edu/cgi/viewcontent.cgi?article $=1067 \&$ context $=$ econ_working_papers.

Preuss, H.,Könecke,T., \& Schütte,N. (2010). Calculating the primary economic impact of a sports clubs regular season competition: A first model. Journal of Sporting Science and Physical Education, 60, 17-22.

Propheter, G. (2012). Are Basketball Arenas Catalysts of Economic Development?. Journal of Urban Affairs, 34 (4), 441-459. https://doi. org/10.1111/j.1467-9906.2011.00597.x.

Pujol, F., \& García-del-Barrio, P. (2006). Informe: El Valor Mediático Del Mundial de Basket 2016. Departamento de Economía, Universidad de Navarra y Universitat International de Catalunya. https://upcommons.upc.edu/bitstream/handle/2099/3914/valor_mediatico.pdf.

Ramírez J.M; Ordaz, J.A, \& Rueda, J.M (2007). Evaluación del impacto económico y social de la celebración de grandes eventos deportivos a nivel local: el caso del Campeonato de Tenis femenino de la ITF en Sevilla en 2006. Revista de métodos cuantitativos para la economía y la empresa, 20-39.

Saayman, M., \& Saayman, A. (2012). Determinants of spending: An evaluation of three major sporting events. International Journal of Tourism Research, 14(2), 124-138. http://dx.doi.org/10.1002/jtr.841.
Salgado, J., Barajas, A., \& Sánchez, P. (2017). Economic impact of sport: Topic of growing interest for the scientific literature. Revista Interna cional de Medicina y Ciencias de la Actividad Física y del Deporte, 17(68). https://doi.org/10.15366/rimcafd2017.68.010.

Salgado, J., Barajas, A., \& Sánchez-Fernández, P. (2018b). Determinants of the spending of sporting tourists: The case of attendees at professional basketball. European Research on Management and Business Economics, 24(3), 168-176. https://doi.org/10.1016/j.iedeen.2018.05.001.

Salgado, J., Sánchez, P., \& Barajas, A. (2020). Differences in the Behavior of Tourist Expenditure According to the Sports Category of the Event: Evidence for a Master and Absolute Championship. In Advances in Tourism, Technology and Smart Systems (pp. 357-367). Springer, Singapore.

Salgado, J., Sánchez, P., Pérez, M., \& Barajas, A. (2018a). Economic valuation of a medium-sized sporting event: impact of the Spanish Swimming Championship. Journal of Physical Education and Sport, 18 1349-1355

Sánchez, P., Salgado, J., Rodríguez, A., \& Barajas, A. (2016). Impacto económico del XXI Campeonato de España “Open” de Invierno Master de Natación de Pontevedra 2015. SPORT TK-Revista EuroAmericana de Ciencias del Deporte, 5, 169-180. https://revistas.um.es/sportk/article/view/254191.

Sánchez, P., Salgado, J., Rodríguez, A., \& Barajas, A. (2016). Impacto económico del XXI Campeonato de España "Open" de Invierno Master de Natación de Pontevedra 2015. SPORT TK-Revista EuroAmericana de Ciencias del Deporte, 5(1) 169-180. https://doi.org/10.6018/254191.

Sato, M., Jordan, J.S., Kaplanidou, K., \& Funk, D.C. (2014). Determinants of tourists' expenditure at mass participant sport events: A five-year analysis. Current Issues in Tourism, 17(9), 763-771. http:// dx.doi.org/10.1080/13683500.2014.892918.

Smith, A. (2010). Leveraging benefits from major events: Maximising opportunities for peripheral urban areas. Managing Leisure, 15(3), 161-180. https://doi.org/10.1080/13606710902752794.

Taks, M., Chalip, L., Green, B. C., Kesenne, S., \& Martyn, S. (2009). Factors affecting repeat visitation and flow-on tourism as sources of event strategy sustainability. Journal of Sport \& Tourism, 14(2-3), 121 142. https://doi.org/10.1080/14775080902965066.

Taks, M., Kesenne, S., Chalip, L., \& Green, C. B. (2011). Economic impact analysis versus cost benefit analysis: The case of a medium-sized sport event. International Journal of Sport Finance, 6(3), 187-203. Recuperado de: https://scholar.uwindsor.ca/cgi/viewcontent.cgi?article $=1028 \&$ context=humankineticspub.

Ziakas, V. (2019). Issues, patterns and strategies in the development of event portfolios: configuring models, design and policy. Journal of Policy Research in Tourism, Leisure and Events, 11(1), 121-158. https://doi. org/10.1080/19407963.2018.1471481.

Ziakas, V., \& Costa, C. (2011a). The Use of an Event Portfolio in Regional Community and Tourism Development: Creating Synergy between Sport and Cultural Events. Journal of Sport \& Tourism, 16 (2), 149175. https://doi.org/10.1080/14775085.2011.568091.

Ziakas, V., \& Costa, C.A. (2011b). Event portfolio and multi-purpose development: Establishing the conceptual grounds. Sport Management Review, 14(4), 409-423. https://doi.org/10.1016/j.smr.2010.09.003. 